\section{Lentiginous eruption in resolving psoriasis plaques during treatment with ixekizumab: a case report and review of the literature}

\author{
Pablo Santa María, ${ }^{1}$ \\ Fernando Valenzuela, ${ }^{1}$ \\ Claudia Morales, ${ }^{2}$ Raul De la Fuente, ${ }^{3}$ \\ Roberto Cullen ${ }^{1}$
}

${ }^{1}$ Dermatology Department, University of Chile Clinical Hospital; ${ }^{2}$ Department of

Pathology, Dermopathology Section,

University of Chile Clinical Hospital;

${ }^{3}$ Faculty of Medicine, University of

Chile, Santiago, Chile

\begin{abstract}
We report the case of a 56-year old male with severe plaque psoriasis that was successfully treated with ixekizumab, a new anti interleukin (IL)-17 monoclonal antibody. During the first months of treatment he developed a lentiginous eruption in the sites of rapidly resolving plaques. Biopsy and immunohistochemistry reports showed elements of both lentigo and post-inflammatory hyper pigmentation. These findings, which have been increasingly described in anti-tumor necrosis factor alpha (TNF $\alpha)$ and anti IL-12/IL-23 therapy, may be explained by the down regulating effect of TNF $\alpha$ and IL-17 on pigmentation genes, which is very rapidly suppressed by ixekizumab, resulting in hyper pigmentation, and by the alteration of mesenchymal-epidermal interaction via keratinocyte growth factor during the inflammatory period, which results in the development of histopathological elements of lentigo.
\end{abstract}

\section{Introduction}

The development of a rare lentiginous eruption in sites of resolving psoriatic plaques has been previously reported. It has been also described as speckled pigmentation and naevus spilus - like pigmentation. The precise mechanism involved is still unclear. Although first described during phototherapy treatment, the development of these lentigines after biological therapy is a phenomenon that has been reported relatively often in the last few years. We present the case of a man who developed such lentigines while being treated with ixekizumab.

\section{Case Report}

A 56-year old male, skin phototype 3 of Fitzpatrick, was admitted in our outpatient clinic with a 10-year history of extensive plaque psoriasis, type 2 diabetes, dyslipidemia and hypertension. He had been previously treated with topical corticosteroids and methotrexate with no significant clinical response. He presented plaque psoriasis with a body surface area (BSA) compromise of $62 \%$ and a psoriasis area and severity index (PASI) of 38.9, consistent with severe plaque psoriasis. He began treatment with ixekizumab, a new anti-interleukin (IL)17A monoclonal antibody, with the following regimen: $160 \mathrm{mg} \mathrm{sc}$. at week 0,80 mg every 2 weeks for the first 3 months and every 4 weeks since. At week 12 of treatment he had developed an $80 \%$ reduction in affected area (BSA) and a $92 \%$ reduction in PASI. 6 months after starting therapy he had no visible psoriasis (PASI 100 response). Between the first and second months of treatment he developed an asymptomatic lentiginous eruption exclusively at the sites of resolving plaques, in the trunk and proximal extremities (Figure 1). Dermoscopy of the pigmented lesions showed a regular reticular pattern (Figure 2). The eruption was interpreted as an atypical post-inflammatory hyperpigmentation, but the unusual speckled pattern persuaded us to take a biopsy to confirm the hypothesis. Biopsy exhibited basal epithelial melanosis, elongated rete ridges (with mild anastomosis), dermal melanophages and a mild perivascular superficial inflammatory infiltration of lymphocytes, consistent with both postinflammatory hyperpigmentation and lentigo (Figure 3A). The immunohistochemical melanocyte markers HMB-45 and S100 showed no significant increase in melanocyte count (Figure 3B). He received no other treatment but was instructed to avoid sun exposure.

\section{Discussion}

Naevus spilus-like pigmentation in resolving psoriasis plaques was initially reported following UVA or UVB phototherapy. UV radiation is a known producer of freckling, so consequently researchers believed the phenomenon to be a form of abnormal reaction to UV light. ${ }^{1}$ However, in the last decade it has been increasingly described after biologic therapy with antitimor necrosis factor alpha (TNF $\alpha$ monoclonal antibodies (infliximab, ${ }^{2}$ adalimumab ${ }^{3}$ and etanercept ${ }^{4}$ ) and ustekinumab ${ }^{5}$ (anti IL12/IL-23 monoclonal antibody), especially
Correspondence: Pablo Santa María, Dermatology Department, University of Chile Clinical Hospital, 999 Santos Dumont, Independencia, Santiago 8380456, Chile. Tel.: +56.22.9788173 - Fax: +56.22.7771142. E-mail: psantamariahighet@gmail.com

Key words: Lentiginous eruption; post inflammatory hyper-pigmentation; psoriasis, ixekizumab.

Contributions: the authors contributed equally.

Conflict of interest: the authors declare no potential conflict of interest.

Received for publication: 6 February 2017. Accepted for publication: 5 September 2017.

This work is licensed under a Creative Commons Attribution-NonCommercial 4.0 International License (CC BY-NC 4.0).

(C) Copyright P. Santa Maria et al., 2017

Licensee PAGEPress, Italy

Dermatology Reports 2017; 9:7079

doi:10.4081/dr.2017.7079

in severe cases that respond rapidly to therapy. Some of those reports have shown an increase of melanin deposition without an increment in melanocyte count or the formation of melanocyte nests, which suggests that this condition emerges from some kind of post-inflammatory melanin production. ${ }^{3,4}$

Post-Inflammatory hyper pigmentation (PIH) is a fairly understood phenomenon that results from the oxidation of arachidonic acid to intermediates that form prostaglandins, leukotrienes and other mediators that in turn stimulate epidermal melanocytes. ${ }^{6}$ Sun exposure increases the problem by the release of reactive oxygen species. PIH occurs more frequently in inflammatory dermatoses that rely on such mediators, like acne vulgaris, allergic reactions, burns and infections. It is especially severe in dermatoses that disrupt the basal cell layer (which results in pigmentary incontinence), such as lichenoid dermatosis and lupus. On the other hand, PIH in psoriasis is less frequent when compared to other epidermal inflammatory conditions, it can develop in all skin phototypes and usually clears out on its own in a few weeks or months after the flare is over. These clinical discrepancies may be explained because $\mathrm{PIH}$ in psoriasis appears to be mediated by different mechanisms. Unlike other skin inflammatory conditions, $\mathrm{PIH}$ in psoriasis is believed to be the result of inflammation driven by TNF $\alpha$ and IL-17, which induce the down-regulation of pigmentation genes such as MC1R, MITF, SOX-10, DCT and $\mathrm{TYR}^{7}$ (In turn, this particular mechanism 
accounts for the common hypo-pigmented spots in resolving plaques). Akamura et al. have recently postulated a melanocyte autoantigen (disintegrin-like and metalloprotease domain containing thrombospondin type 1 motif-like 5, ADAMTSL5) as a potential target for auto-reactive T-cells in psoriatic skin. ${ }^{8}$ The degranulation of IL-17driven Th1 cells in the presence of melanocytes could be responsible for this interference on melanogenesis (Interestingly, INFy-driven Th1 attack on melanocytes, as in the case of vitiligo, results in melanocyte death, not just downregulation). During this inflammatory period, melanocyte growth-promoting factors such as CXCL1, CXCL2, CXCL3 and IL-8 are increased in order to compensate for the down-regulated melanocytes ${ }^{7}$. Once the flare is over, dormant melanocytes are suddenly over-stimulated by pro-pigmentation mediators, resulting in PIH.

Although both TNF $\alpha$ and IL-17 have been associated with the down-regulation of pigmentation genes, the effect is more pronounced in IL-17. A recent report by Wang et al. showed that anti IL-17 (ixekizumab) therapy resulted in a considerably faster recovery of pigmentation signaling levels when compared with anti TNF $\alpha$ treatment (etanercept) in psoriasis lesional skin. ${ }^{7}$

Additionally, PIH does not usually show dermoscopic elements of lentigo (i.e., regular reticular pattern), nor does it develop histopathological findings of lentigo, such as elongated and anastomosed dermal papillae. Increased levels of KGF, a mesenchymal-epidermal cross-talk mediator known to play a role in wound healing and hair follicle development, ${ }^{9}$ may explain these findings. KGF in the dermis and its epithelial receptor (KGFr) are elevated during the inflammatory stages of psoriasis and during the initiation of solar lentigos ${ }^{6,9}$, In addition, KGF has been found to induce both melanin deposition and the development and elongation of rete ridges in vivo. ${ }^{9}$

\section{Conclusions}

We postulate that these recent advances in the understanding of psoriasis-associated hyper pigmentation and the initiation of solar lentigos offer a plausible explanation for the development of this rare eruption.

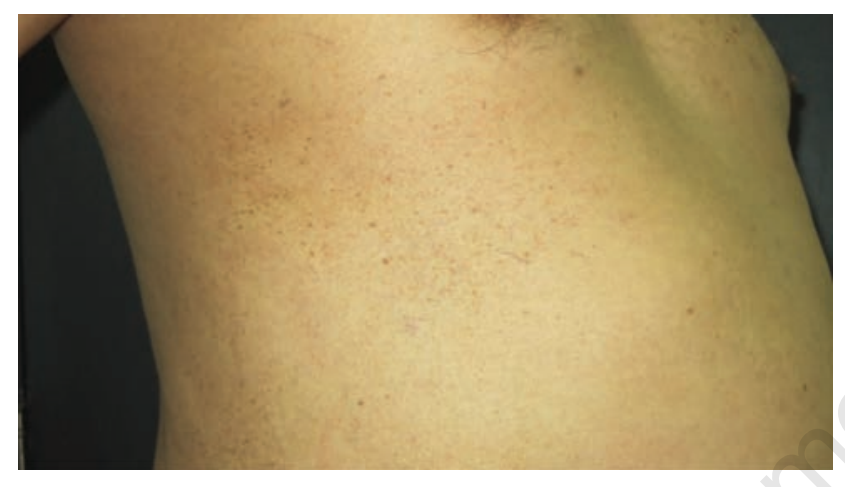

Figure 1. Naevus spilus-like lentiginous eruption developing on the site of a resolved psoriasis plaque.

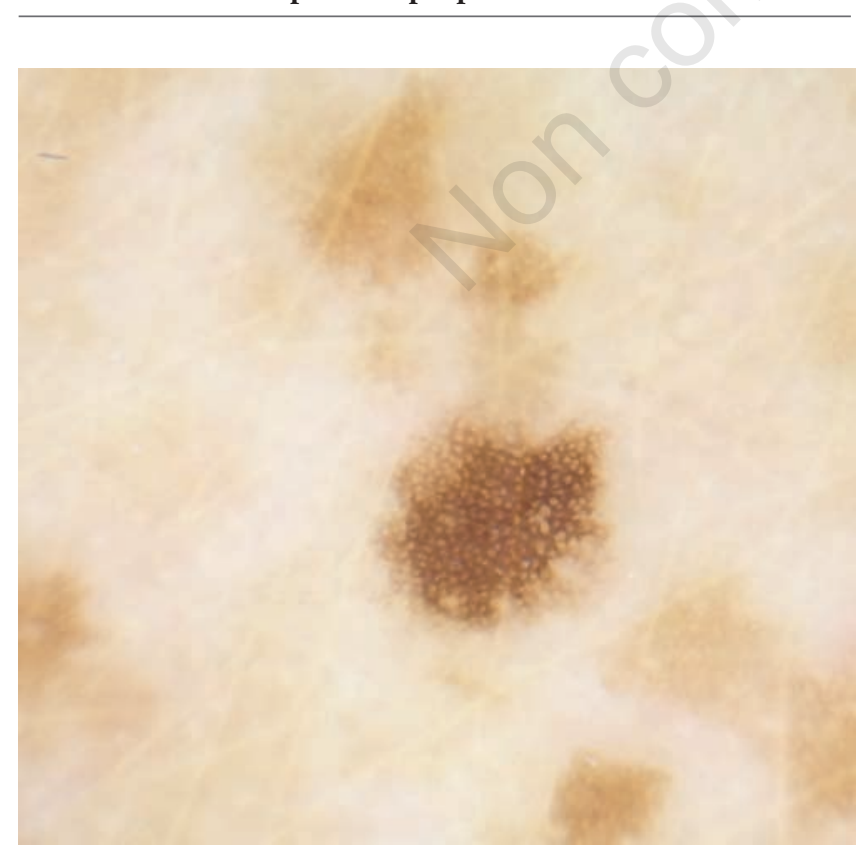

Figure 2. Dermoscopy of the lentiginous eruption, showing a regular reticular pattern consistent with lentigo
A



B

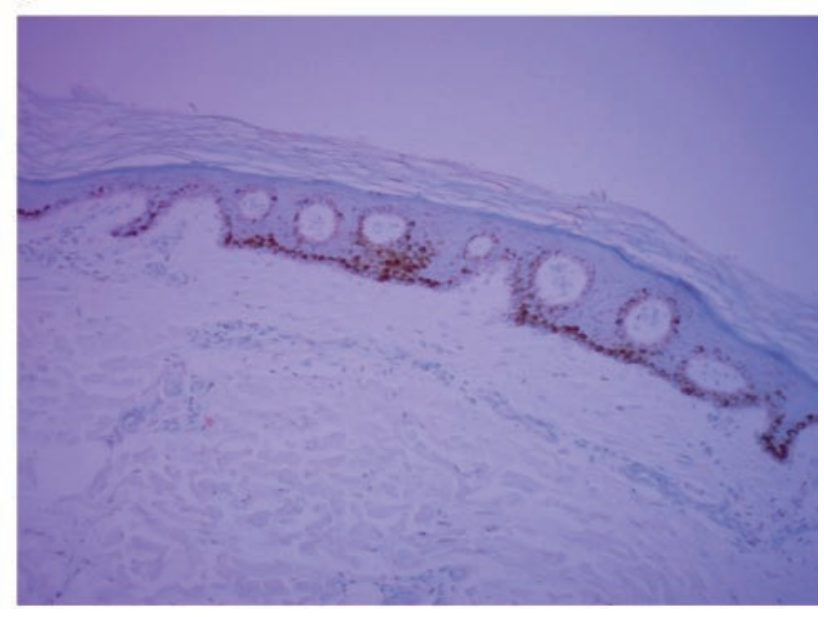

Figure 3. A) Skin biopsy of the lentigines, exhibiting increased melanin deposition in the basal epidermal layer, acantosis and joined papillae (hematoxylin and eosin, original magnification, $x$ 40); B) Immunohistochemical analysis, showing basal melanine deposition and a normal melanocyte count. (HMB45, original magnification, $x$ 40). 
Furthermore, the role that IL-17 plays in such instances leads us to believe that the use of IL-17 inhibitors in the coming years will be increasingly associated with the appearance of lentiginous eruptions in resolving plaques, even more so than antiTNF $\alpha$.

\section{References}

1. Burrows NP, Handfield-Jones S, Monk $\mathrm{BE}$, et al. Multiple lentigines confined to psoriatic plaques. Clin Exp Dermatol 1994;19:380-2.

2. Dogan S, Atakan N. Multiple lentigines confined to psoriatic plaques induced by biologic agents in psoriasis therapy: a case and review of the literature. Cutan
Ocul Toxicol 2015;34:262-4.

3. Santos-Juanes J, Coto P, Mallo S, et al. Multiple lentigines confined to resolving psoriatic plaques in a patient treated with adalimumab. Dermatology 2008;216:279.

4. Costa LA, Belinchón I, Betlloch I, et al. Multiple lentigines arising in resolving psoriatic plaques after treatment with etanercept. Dermatol Online J 2008;14:11.

5. Gutiérrez-González E, Batalla A, de la Mano D. Multiple lentigines in areas of resolving psoriatic plaques after ustekinumab therapy. Dermatol Online J 2014;20:22338.

6. Cardinali G, Kovacs D, Picardo M. Mechanisms underlying post-inflamma- tory hyperpigmentation: lessons from solar lentigo. Ann Dermatol Venereol 2012;139:S148-52.

7. Wang CQ, Akalu YT, Suarez-Farinas $\mathrm{M}$, et al. IL-17 and TNF synergistically modulate cytokine expression while suppressing melanogenesis: potential relevance to psoriasis. $\mathrm{J}$ Invest Dermatol 2013;133:2741-52.

8. Arakawa A, Siewert K, Stöhr J et al. Melanocyte antigen triggers autoimmunity in human psoraisis. J Exp Med. 2015;212:2203-12.

9. Chen N, Hu Y, Li WH, et al. The role of keratinocyte growth factor in melanogenesis: a possible mechanism for the initiation of solar lentigines. Exp Dermatol 2010;19:865-72. 